\title{
AN ADDITIONAL HAZARD OF ENDOTRACHEAL INTUBATION
}

\author{
George W. Seuffert, M.D., and Karl F. Urbach, M.D., Ph.D.*
}

IT IS WELL KNOWN that the cuff of an endotracheal tube may be distorted by inflation and produce airway obstruction. The following case illustrates an unusual variation of this complication.

The patient was a healthy 37-year-old male who was anaesthetized for varicose vein stripping and ligation of the left leg. Following a thiopental induction, ether was added to the anaesthesia. Persistent laryngospasm and copious secretions made maintenance of his airway difficult. A $9.5 \mathrm{~mm}$. endotracheal tube was inserted into the trachea with the aid of relaxation by succinylcholine. The cuff was checked rapidly for leaks just prior to intubation. The intubation was technically difficult. It was necessary to inject $8 \mathrm{ml}$. of air before a gas-tight system was established. Good breath sounds were heard throughout the chest.

Over the next hour, it was necessary to inject a total of $8 \mathrm{ml}$. of air in $1-3 \mathrm{ml}$. increments to maintain a gas-tight fit. It was thought that a small leak was present in the cuff. At this time, the patient coughed on the tube and developed bronchospasm and paradoxical respiration. The cuff was deflated and the tube withdrawn approximately $2 \mathrm{~cm}$. There was a slight leak between the endotracheal tube and the trachea at this time. Endotracheal suction had no effect on the respiration. There were breath sounds in both lungs. The cuff was reinflated with $8 \mathrm{ml}$. of air and it was possible to ventilate the patient adequately, using pressures of less than $25 \mathrm{~cm}$. of water. Anaesthesia was resumed until surgery ended 15 minutes later.

Paradoxical respiration returned when the patient breathed spontaneously through the endotracheal tube. After the completion of surgery, ventilation was assisted for 10 minutes. The average tidal volume was $450 \mathrm{ml}$. At this time, the patient reacted to the tube and moved his extremities. After apparent deflation of the cuff, the endotracheal tube was removed and a herniated cuff was discovered (see Fig. 3). The paradoxical respiration disappeared immediately.

Postoperatively, the patient complained of a sore throat for two days. This responded to symptomatic therapy. Indirect laryngoscopy performed one week later revealed no pathology.

\section{Discussion}

This problem originated when the double-walled cuff was incorrectly applied to the endotracheal tube. The inner wall slipped beneath the constricting band of the cuff as it was fitted over the tube. When the cuff was inflated with a low volume and pressure, it appeared to function properly (Fig. 1).

'Dr. Urbach is Chief, and Dr. Seuffert is Deputy Chief, Anesthesiology Service, U.S. Public Health Service Hospital, Staten Island, New York. 


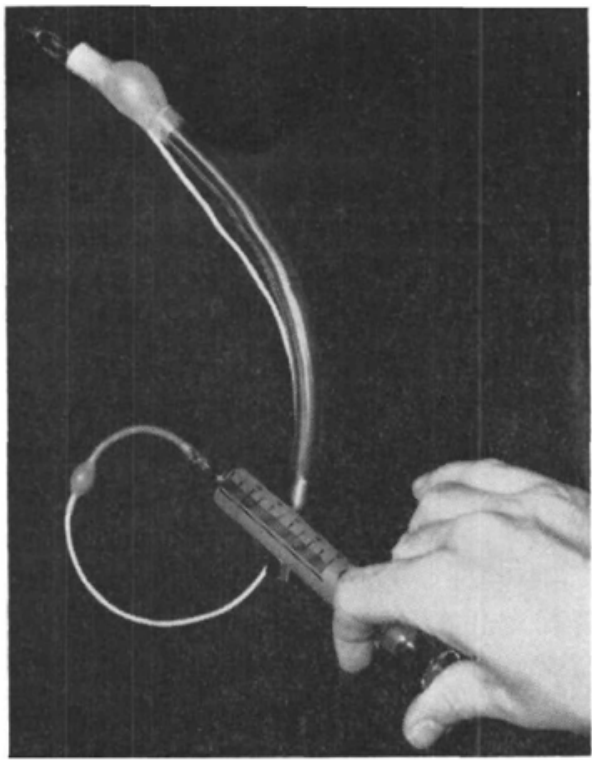

Figure 1. Cuff inflated with low pressure.

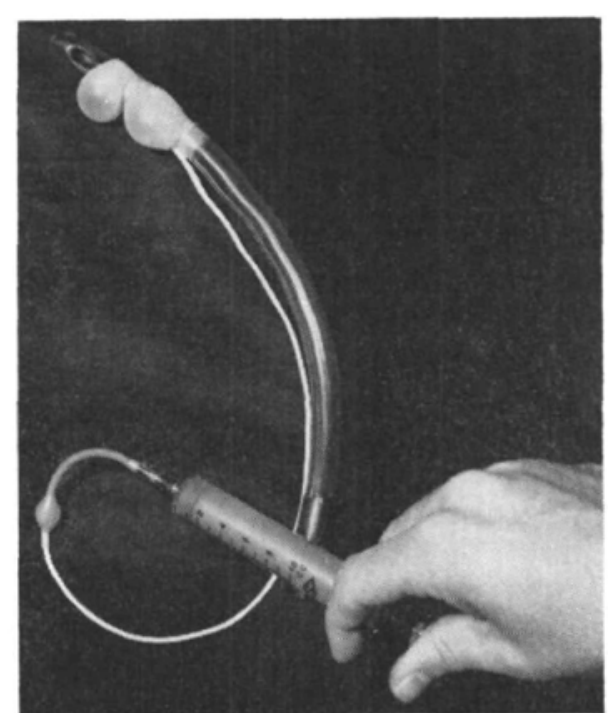

Figure 2. Probable appearance in trachea.

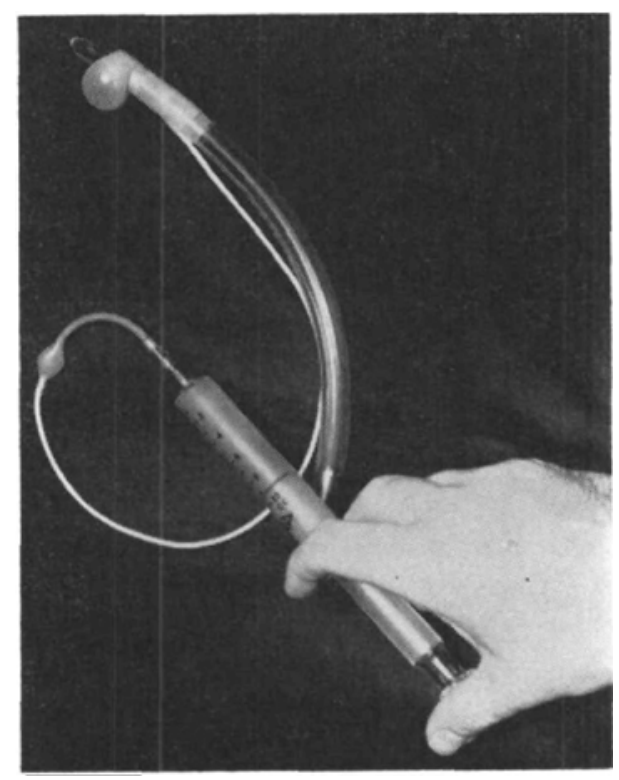

FIGURE 3. Appearance of cuff after extubation.

A greater volume of air injected into the cuff to provide a gas-tight seal resulted in herniation of the inner wall (Fig. 2). This herniation probably forced the bevel of the tube against the trachea to produce partial airway obstruction. When the air was released from the cuff, the shoulder of the cuff acted as a one way valve and prevented the herniation from emptying (Fig. 3). 